\title{
La Modelización Molecular como Herramienta para el Diseño de Nuevos Polímeros Conductores
}

\author{
Jordi Casanovas \\ Departament de Química, Universitat de Lleida, Spain \\ Elaine Armelin, José Ignacio Iribarren, Carlos Alemán \\ Departament d'Enginyeria Química, Universitat Politècnica de Catalunya, Spain \\ Francisco Liesa \\ Departament d'Enginyeria Mecànica, Universitat Politècnica de Catalunya, Spain
}

\begin{abstract}
Resumen: Se presenta la capacidad de las técnicas de modelización molecular basadas en métodos de la química cuántica para predecir la estructura molecular y electrónica de polímeros conductores. Concretamente, se discute la aplicabilidad de estas herramientas computacionales al estudio de diferentes aspectos del politiofeno y sus derivados: geometría molecular y planaridad, cambios estructurales producidos por el dopado, propiedades electrónicas y desarrollo de nuevos materiales conductores.
\end{abstract}

Palabras clave: Polímero conductor, politiofeno, modelización molecular, química cuántica, estructura electrónica, estructura molecular.

\section{Molecular Modeling Tools to Design New Conducting Polymers}

Abstract: The ability of molecular modeling techniques based on quantum chemical methods to predict the molecular and electronic structure of organic conducting polymers is examined. More specifically, we report on the applicability of these computational tools to study different aspects of polythiophene and its derivatives: molecular geometry and planarity, the structural changes induced by the doping process, the electronic properties and the design of new conducting materials.

Keywords: Conducting polymer, polythiophene, molecular modeling, quantum chemistry, electronic structure, molecular structure.

\section{Introducción}

La historia nos ha mostrado más de una vez que un error humano, o la fortuna, puede ser el punto de partida de un gran avance científico. A principios de los años 70, un estudiante del profesor $\mathrm{H}$. Shirakawa estaba realizando la síntesis de poliacetileno a partir de acetileno gaseoso. Durante el proceso de polimerización añadió mucho más catalizador del que era necesario, lo que provocó cambios importantes en la estructura del polímero, dando lugar a un producto que presentaba una conductividad eléctrica inusualmente elevada. Pocos años después, Shirakawa, MacDiardmid y Heeger publicaron dos artículos en los que mostraban que la conductividad del poliacetileno podía aumentar mil millones de veces al doparlo con vapores de yodo ${ }^{[1]}$. El descubrimiento de los polímeros conductores les valió el Premio Nóbel de Química del año 2000 y fue el precursor de la síntesis de más de un centenar de polímeros capaces de conducir la corriente eléctrica.

El hallazgo de los polímeros conductores, también denominados metales sintéticos, cambió de forma radical la visión que se tenía de los materiales poliméricos y, en particular, sus posibles aplicaciones. Para tomar conciencia de ello, basta con pensar que la utilización de polímeros conductores permite combinar, en un sólo material, las propiedades eléctricas de los conductores metálicos clásicos con las múltiples ventajas de los plásticos-inercia química, baja densidad, bajo coste, procesabilidad-. Esta posibilidad ha llevado incluso a la sustitución de piezas metálicas por componentes poliméricos en muchas aplicaciones.

En los últimos años se ha intensificado de forma especial la investigación en polímeros conductores heterocíclicos, que pueden ser sintetizados tanto química como electroquímicamente ${ }^{[2]}$, ya que a ellos pueden agregarse distintos grupos funcionales que permiten regular fácilmente sus propiedades eléctricas, ópticas y magnéticas ${ }^{[3]}$. Entre los polímeros heterocíclicos destacan los polímeros basados en anillos de pirrol, furano y tiofeno (Esquema 1), pues poseen una estabilidad ambiental excelente además de buenas propiedades eléctricas y ópticas ${ }^{[3,4]}$. Por ello, sus aplicaciones actuales son numerosas: baterías orgánicas, visores electrocrómicos, sensores químicos, diodos emisores de luz

Autor para correspondencia: Carlos Alemán, Departament d'Enginyeria Química, ETSEIB, Universitat Politècnica de Catalunya, Avda. Diagonal 647, Barcelona E-08028, Spain. E-mail: carlos.aleman@upc.edu. Jordi Casanovas, Departament de Química, Escola Politécnica Superior, Universitat de Lleida, c/ Jaume II n69, Lleida E-2500, Spain.E-mail: jcasanovas@quimica.udl.es 


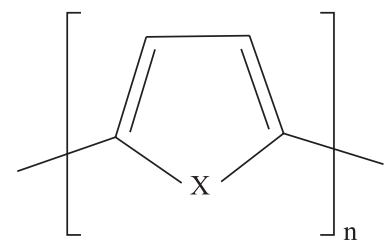

Esquema 1. Polímeros heterocíclicos: polipirrol $(X=N H)$, polifurano $(X=O)$ y politiofeno $(\mathrm{X}=\mathrm{S})$.

(LED), antiestáticos, emisores de interferencias electromagnéticas y bloqueadores de radares, membranas para la depuración de agua, etc. Sin embargo, las aplicaciones más revolucionarias las encontramos en el campo de la biomedicina ${ }^{5}$, entre las que podemos destacar su utilización en la ingeniería de tejidos, prótesis neurológicas y musculares, el diseño de biosensores para la determinación de glucosa, los sistemas liberadores de fármacos y la biomecánica.

La investigación de polímeros conductores se lleva a cabo, tanto en ámbitos académicos como industriales, desde enfoques tan distintos como la química sintética, la electroquímica, la física del estado sólido, la química-física, la ciencia de los materiales o la ingeniería de componentes. La modelización molecular basada en métodos de la química cuántica, también denominada química computacional, constituye una herramienta poderosa que hoy en día contribuye de forma eficaz al desarrollo de nuevos materiales. Esta herramienta permite predecir, por ejemplo, la estructura química, las propiedades y la reactividad de un producto antes de que sea sintetizado. Por ello se utiliza de forma regular para complementar, interpretar, predecir o incluso sustituir medidas experimentales, con el consiguiente ahorro de tiempo, material y laboratorios especializados, a la vez que simplifica de forma considerable el trabajo de personal altamente cualificado. En consecuencia, encontramos aplicaciones de la modelización molecular en ámbitos diversos como son la industria farmacéutica (diseño de fármacos), la industria química (diseño de catalizadores y de procesos químicos, mejora de mecanismos de reacción, formulación de nuevos productos) o la química de la atmósfera.

En este artículo analizamos el papel que puede jugar la modelización molecular en el desarrollo de los polímeros conductores. Como modelo de estudio usamos distintos polímeros de la familia de los politiofenos, pues poseen una estructura molecular simple pero un gran potencial de aplicación $^{[2,3]}$.

\section{La conducción eléctrica en polímeros}

Polímeros aislantes. El fenómeno de la conducción eléctrica implica el movimiento de partículas con carga eléctrica normalmente, electrones- en el interior de un material, cuando sobre él se aplica un campo eléctrico. En un polímero, llamémosle "convencional", los electrones de valencia están formando enlaces químicos entre los átomos y prácticamente no tienen libertad de movimiento. Esta visión tan simple permite comprender porqué la gran mayoría de polímeros son, en condiciones normales, aislantes eléctricos. Desde el punto de vista de la Teoría de Bandas -la teoría que hoy en día explica mejor el comportamiento eléctrico de los materiales-, las propiedades eléctricas de una sustancia vienen determinadas por su estructura de bandas. Y más concretamente, por la diferencia de energía que existe entre su banda de valencia (formada por los orbitales que contienen los electrones de mayor energía) y su banda de conducción (constituida por los orbitales vacíos de menor energía). En los polímeros convencionales, la diferencia de energía entre ambas bandas ("band gap" o $\mathrm{E}_{\mathrm{g}}$ ) es grande, en general mayor que $2.0 \mathrm{eV}$ (Figura 1a). Cuando a temperaturas normales se aplica un campo eléctrico sobre el polímero, la energía adicional que adquieren sus electrones no es suficiente como para que éstos puedan pasar de la banda de valencia a la banda de conducción. En consecuencia, los electrones no pueden moverse libremente por el interior del material. Nos referimos entonces a un polímero aislante.

Polímeros conductores. Los polímeros conductores más comunes presentan una distribución alterna de enlaces carbonocarbono simples y dobles a lo largo de sus moléculas. Este hecho permite una deslocalización considerable de los electrones de valencia a lo largo del sistema $\pi$ de la cadena polimérica. Sin embargo, esta deslocalización no suele ser suficiente como para que el material sea conductor. El polímero neutro con estas características puede convertirse en conductor si se hace reaccionar con un agente oxidante (o con uno reductor). El proceso que tiene lugar es una reacción redox entre las cadenas poliméricas y los aceptores (o los dadores) de electrones. En el lenguaje de la física del estado sólido, el proceso se denomina dopado, por analogía con la síntesis de semiconductores extrínsecos inorgánicos: la utilización de un agente oxidante corresponde a un dopado de tipo $\mathrm{p}$, mientras que el uso de un agente reductor implica un dopado de tipo $\mathrm{n}$. En consecuencia, un criterio a tener en cuenta en la selección de un polímero con posibilidades conductoras es su facilidad para oxidarse o reducirse. La utilización de polímeros heterocíclicos, como los politiofenos, permite trabajar con materiales de bajo potencial de ionización (fácil oxidación) y/ o alta afinidad electrónica (fácil reducción).

Desde el punto de vista de la Teoría de Bandas, la oxidación o la reducción de las moléculas poliméricas causa la aparición de estados electrónicos situados en el band gap, que facilitan el proceso de conducción ${ }^{[6]}$. En el caso de la oxidación, la eliminación de un electrón de la cadena polimérica hace que el último orbital de la banda de valencia (denominado HOMO: Highest Occupied Molecular Orbital) aumente de energía, a la vez que el orbital de conducción de menor energía (denominado LUMO: Lowest Unoccupied Molecular Orbital) se estabiliza. Ello conlleva la aparición de dos estados situados en el band gap (Figura 1b). Se forma lo que, en física del estado sólido, se denomina un polarón (o un polarón positivo). En el lenguaje químico es un catión radical. Si se arranca un segundo electrón de la cadena polimérica, se forma un bipolarón (bipolarón positivo), lo que en terminología química es un dicatión. El bipolarón formado en el dopado p implica la existencia de dos estados electrónicos vacíos en el band gap (Figura 1c). Al aumentar el nivel de dopado, se forman más estados bipolarónicos que se solapan dando lugar a bandas bipolarónicas en el gap (Figura 1d). En el dopado de tipo n, se 


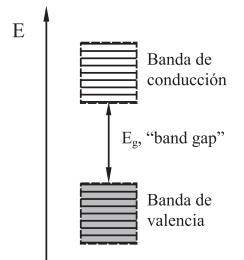

(a)

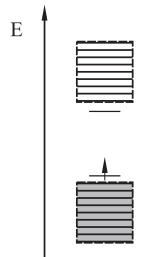

(b)

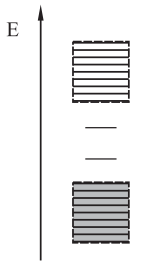

(c)

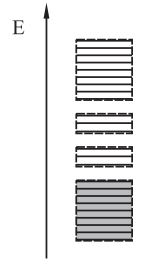

(d)
Figura 1. Representación esquemática de la estructura de bandas de: (a) un polímero neutro aislante; (b) un polímero con un polarón; (c) un polímero con un bipolarón; y (d) un polímero con bandas bipolarónicas.

pueden formar polarones y bipolarones negativos, estando los estados electrónicos del bipolarón ocupados por electrones. El politiofeno dopado posee una conductividad eléctrica muy superior a la del polímero no dopado. Ello es porqué, gracias a la presencia de polarones y bipolarones, algunos electrones del politiofeno dopado quedan "libres", al poder abandonar la banda de valencia mediante transiciones electrónicas que requieren una energía inferior al valor del band gap. Este hecho puede ser comprobado mediante espectroscopia de absorción óptica o mediante técnicas electroquímicas ${ }^{[7]}$. Por otra parte, es importante señalar que la conductividad de los politiofenos puede ser controlada a través de la concentración de agente dopante, siendo también destacable la reversibilidad electroquímica que existe entre los estados neutro y dopado.

El dopado de un polímero conjugado conlleva, además de la alteración descrita de su estructura electrónica, cambios importantes en su geometría. Siguiendo con el ejemplo del politiofeno neutro, sabemos que su geometría más estable corresponde a una estructura aromática que se extiende a lo largo de toda la cadena polimérica. Sin embargo, la formación de polarones y bipolarones positivos -es decir, de cationes radicales y de dicationes, respectivamente- implica ciertas distorsiones de las cadenas, que culminan con la transformación hacia una estructura quinoidea ${ }^{[8]}$. La Figura 2 ayuda a comprender este cambio, aunque deben precisarse dos aspectos: (i) en realidad, tanto las cargas como los electrones desapareados no se encuentran localizados sobre los átomos, sino que están repartidos entre un cierto número de anillos; y (ii) hay que tener en cuenta que tanto los electrones como las cargas tienen la facultad de moverse a lo largo de la cadena polimérica.

\section{Modelización molecular}

Las técnicas computacionales de modelización molecular

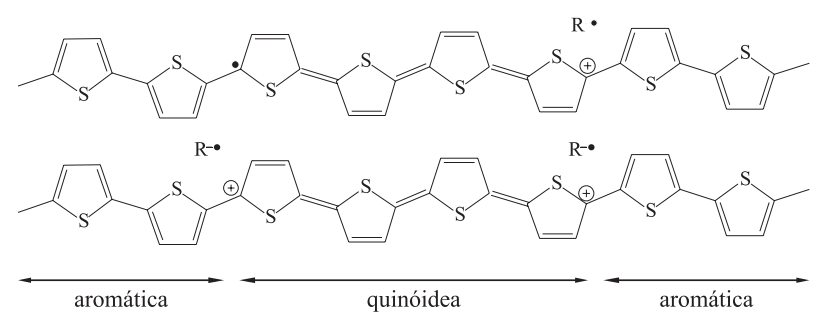

Figura 2. Representación esquemática de una cadena de politiofeno con un polarón (a) y con un bipolarón. (b) $\mathrm{R}^{-}$representa el contra-ion formado en la reacción redox entre la cadena polimérica y el agente dopante aceptor de electrones. permiten crear modelos químicos de materiales diversos, ya sea a nivel molecular o atomístico, mediante la resolución de la ecuación de Schrödinger que describe el comportamiento de los núcleos y los electrones de un sistema. La modelización proporciona, por ejemplo, la geometría más estable, la energía, la distribución de cargas eléctricas o diferentes propiedades espectroscópicas (IR, Raman, NMR, UV-Vis, EPR) del sistema que se está estudiando. Asimismo, permite obtener datos termodinámicos y cinéticos ( como calores de formación y de reacción, entropías, capacidades caloríficas y constantes de velocidad de una reacción química) o propiedades mecánicas (módulos elásticos, curvas de tensión-deformación, etc.).

A continuación se revisan algunos de los resultados que hemos obtenido empleando técnicas de modelización molecular para determinar varias propiedades del politiofeno y de algunos de sus derivados. El objetivo de estos estudios es doble: por un lado, explorar qué puede aportar la modelización molecular al estudio de los polímeros conductores; y, por el otro, comprender mejor el significado de los numerosos datos experimentales que existen actualmente en esta área de investigación. Los resultados que se exponen fueron obtenidos utilizando los paquetes de programas PC-Gamess ${ }^{[9]}$ y Gaussian $98^{[10]}$.

Planaridad. Una de las características principales de los polímeros heterocíclicos conductores es su tendencia a adoptar una geometría plana, pues ésta favorece el máximo solapamiento entre los orbitales atómicos $p$ (conjugación $\pi$ ) $\mathrm{y}$, como consecuencia, la existencia de bandas dadoras de alta energía y/o bandas aceptoras de baja energía. En los poliheterociclos que contienen átomos de azufre la extensión de la conjugación $\pi-\pi$ entre anillos adyacentes es considerable, a la vez que responsable de sus propiedades eléctricas y ópticas. Sin embargo, la posible distorsión con respecto a la conformación anti del ángulo que define la orientación relativa entre anillos adyacentes y la consecuente perdida de planaridad molecular (defecto torsional) afectarían a la estructura electrónica del polímero, llegando incluso a determinar su utilidad. Es por ello que, conforme pasan los años y se incrementa la potencia de los ordenadores, se van sofisticando los métodos de cálculo para poder determinar con la mayor precisión posible los potenciales de torsión ${ }^{[11]}$.

Una primera aproximación al conocimiento de las preferencias conformacionales del politiofeno, y de algunos de sus derivados, puede conseguirse estudiando un compuesto muy simple, el 2,2'-bitiofeno (conocido tanto en fase gas como en estado sólido), y sus derivados dimetílicos. La geometría y el perfil rotacional de estos compuestos fueron analizados mediante cálculos $a b$ initio de mecánica cuántica ${ }^{[12]}$, obteniendo resultados que concuerdan perfectamente con los datos experimentales disponibles. Más recientemente, extendimos el estudio a oligómeros de tiofeno de mayor tamaño ${ }^{[13,14]}$. Como ejemplo de este tipo de estudios, en la Figura 3 se representa el perfil rotacional teórico del 2,2'-bitiofeno, obtenido a nivel de cálculo B3PW91/6$31+\mathrm{G}(\mathrm{d}, \mathrm{p})^{[15]}$, donde se puede apreciar la presencia de dos conformaciones de energía mínima ${ }^{[16]}$. Estos resultados, que 


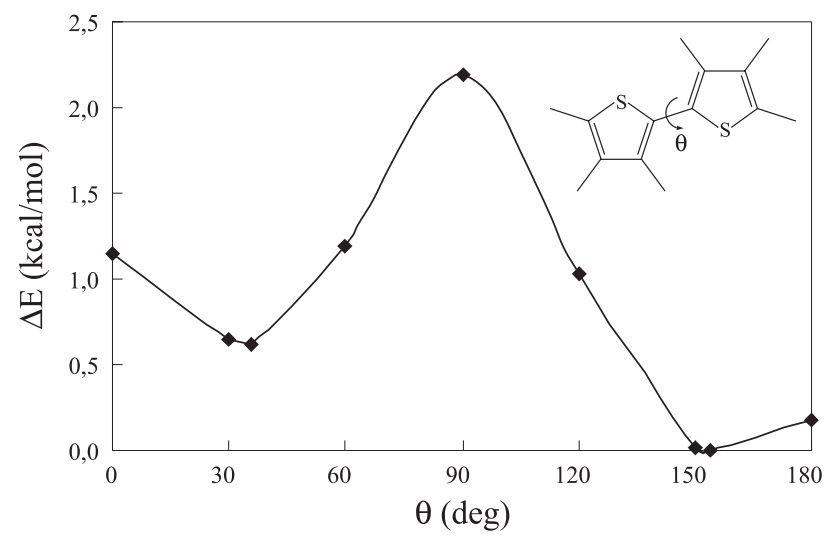

Figura 3. Modelización teórica del perfil rotacional del 2,2'-bitiofeno. Los experimentos de difracción de electrones del bitiofeno ${ }^{[17]}$ indican la existencia de dos conformaciones estables con ángulos diedros $\theta=36 \pm 5^{\circ}$ y $\theta=148 \pm 3^{\circ}$.

están en clara sintonía con las observaciones experimentales, se puede relacionar directamente con la extensión de la conjugación efectiva a lo largo de la cadena de polímero (policonjugación).

Proceso de dopado: Potencial de Ionización y Afinidad Electrónica. Tal y como se comentó anteriormente, el dopado del polímero implica su interacción con un agente dopante y la transferencia de electrones desde el polímero hacia el dopante o viceversa. Por ello, es importante poder estimar correctamente el potencial de ionización (PI) y la afinidad electrónica (AE) del polímero que interesa estudiar. El valor del PI indica si un agente dopante aceptor concreto es capaz de ionizar, al menos parcialmente, el polímero. Por otra parte, la AE cobra importancia en el proceso de dopado dador de electrones.

En el ámbito de la modelización molecular se puede hacer una primera estimación del PI utilizando el teorema de Koopman's, es decir, asociando el PI con la energía del HOMO de nuestro modelo ${ }^{[18]}$. De forma similar, se correlaciona la AE con la energía del LUMO. Aunque ambos valores teóricos suelen reproducir correctamente las medidas experimentales, se pueden obtener valores más precisos tomando la propia definición de PI y AE, es decir, evaluando la diferencia energética entre el estado neutro y los estado cargados eléctricamente ${ }^{[19]}$. Por otra parte, se puede tener una estimación del valor de $\mathrm{E}_{\mathrm{g}}$ mediante la diferencia de energías PI-AE. Esta magnitud da una idea de las posibilidades conductoras del polímero, pues representa la energía de la transición electrónica más favorable.

Los valores de $E_{g}$, PI y AE pueden ser calculados no sólo en modelos reducidos, sino también en auténticas cadenas de polímero generadas mediante la aplicación de condiciones periódicas de contorno a su unidad estructural repetitiva. La utilización de esta técnica, que en principio permite reproducir las características de una molécula de polímero de longitud infinita, está limitada a moléculas en las que no hay ningún tipo de alteración o distorsión molecular, ya que de otro modo ésta se repetiría de forma periódica a lo largo de la cadena. La consideración de un sistema molecular periódico proporciona, lógicamente, una descripción más precisa de un polímero que un modelo reducido. Sin embargo, este último suministra resultados muy razonables desde un punto de vista cualitativo $\mathrm{y}$, además, permite analizar de forma precisa la influencia de todo tipo de alteraciones y defectos puntuales en la molécula.

Proceso de dopado: cambios estructurales. Como se ha comentado, es de esperar que el proceso de dopado dé lugar a modificaciones geométricas importantes de las cadenas poliméricas. Lamentablemente, la estructura amorfa que caracteriza a la mayoría de los polímeros complica de forma considerable el estudio experimental de dichos cambios. Desde el punto de vista teórico, se puede modelizar separadamente la cadena polimérica neutra y la eléctricamente cargada, para después analizar las diferencias estructurales entre ambas. De esta manera, pudimos comprobar que la estructura electrónica del 2,2'-bitiofeno y la del 2,2',5',2'-tertiofeno son aromáticas, mientras que sus respectivos dicationes presentan una distribución de electrones con carácter quinoideo ${ }^{[13,20]}$. Posteriormente, con la intención de acercarnos más a las características reales del politiofeno, utilizamos modelos de mayor tamaño que contenían hasta 13 anillos de tiofeno ${ }^{[21]}$. El patrón alterno de distancias $\mathrm{C}-\mathrm{C}$ y $\mathrm{C}=\mathrm{C}$ reveló una estructura claramente benzoidea en la cadena de politiofeno neutro y una distribución quinoidea en la cadena dicatiónica de los oligómeros de tiofeno. Los resultados confirmaron los estudios anteriores, a la vez que permitieron determinar la extensión del defecto bipolarónico. Como ejemplo, la Figura 4 muestra la magnitud de las distancias de enlace C-C obtenidas al modelizar una cadena formada por 6 anillos de tiofeno en una conformación anti $\left(\theta=180^{\circ}\right)$. Los cálculos fueron realizados a nivel HF/6-31G(d), tanto en el caso de la cadena neutra ${ }^{[14]}$ como para la cadena dicatiónica ${ }^{[16]}$. Los patrones complementarios que se observan en la zona correspondiente a los anillos centrales reflejan cómo se intercambian los enlaces $\mathrm{C}-\mathrm{C}$ y $\mathrm{C}=\mathrm{C}$ al oxidar la molécula.

Otra estrategia para investigar los cambios estructurales consiste en modelizar explícitamente la interacción entre el
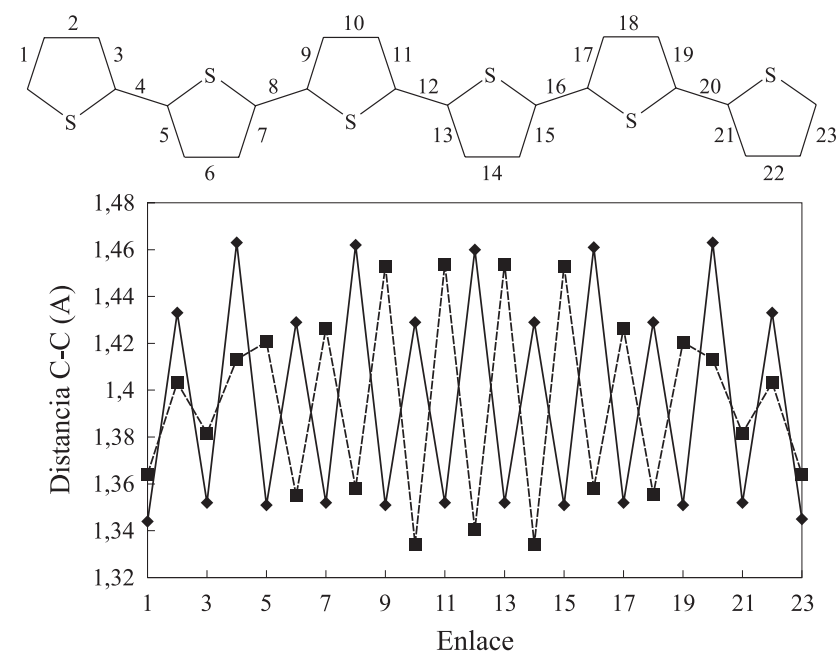

Figura 4. Longitud de los enlaces C-C en el modelo de politiofeno con 6 anillos. La línea continua corresponde a la cadena neutra y la discontinua al dicatión (defecto bipolarónico). 

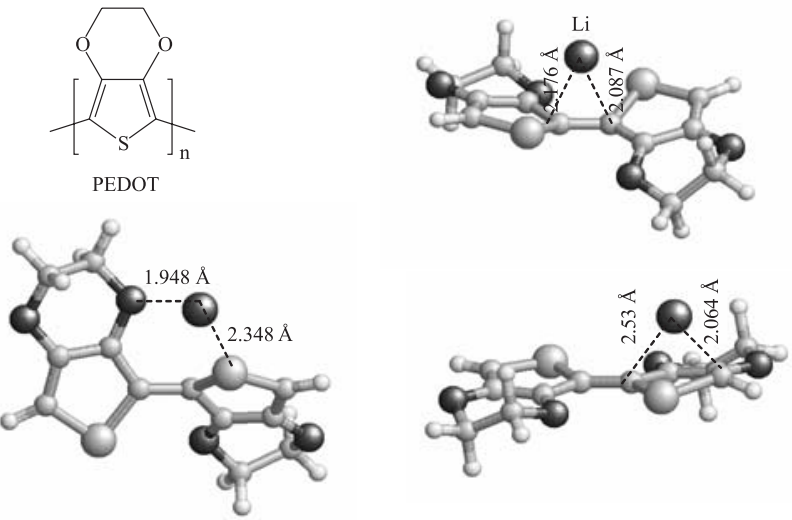

Figura 5. Estructura de los complejos de transferencia de carga Li/2-EDOT.

polímero y el agente dopante, en lugar de analizar únicamente la geometría de los estados inicial (neutro) y final (iónico) del polímero. De esta manera, el estudio incluye información sobre la zona del polímero en la que se produce la interacción con el dopante, así como los efectos de polarización que el dopante ejerce sobre el polímero. Además, la modelización del complejo agente dopante/polímero permite cuantificar la transferencia de carga que tiene lugar durante la interacción. A modo de ejemplo, la Figura 5 muestra la estructura obtenida al modelizar, a nivel UB3PW91/6-31+G(d,p), la interacción de un átomo de $\mathrm{Li}$ con un dímero de 3,4-etilendioxitiofeno (2-EDOT) en tres posiciones distintas ${ }^{[22]}$. Los cálculos realizados muestran el patrón quinoideo en las longitudes de enlace C-C, a la vez que revelan una gran influencia del contra-ion sobre la geometría del oligómero. Como se puede observar en la Figura 5, el átomo de Li es capaz de distorsionar no sólo la conformación anti de las unidades de EDOT consecutivas, sino también la planaridad de los anillos de tiofeno.

Nuevos Materiales. Sin duda, una de las grandes ventajas de la modelización molecular frente a los métodos puramente experimentales es que es posible "imaginar" nuevos materiales y evaluar muchas de sus propiedades sin necesidad de sintetizarlos, con el consiguiente ahorro que ello representa. Por ejemplo, se puede pensar en aumentar la conductividad eléctrica de algún polímero modificando su constitución química o su estructura. En este sentido, recientemente hemos iniciado el estudio teórico de las propiedades estructurales y electrónicas de cinco compuestos que se obtendrían al cambiar los heteroátomos del PEDOT (Esquema 2) ${ }^{[19 a]}$. El estudio sugiere que el compuesto (3), obtenido al intercambiar las

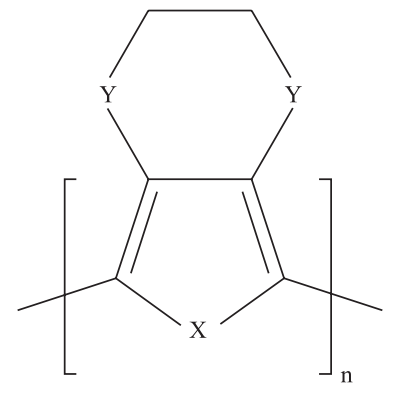

(1) $\mathrm{X}=\mathrm{S}, \mathrm{Y}=\mathrm{O}(\mathrm{PEDOT})$

(2) $\mathrm{X}=\mathrm{Y}=\mathrm{O}$

(3) $\mathrm{X}=\mathrm{O}, \mathrm{Y}=\mathrm{S}$

(4) $X=Y=S$

(5) $\mathrm{X}=\mathrm{N}-\mathrm{H}, \mathrm{Y}=\mathrm{O}$

(6) $\mathrm{X}=\mathrm{N}-\mathrm{H}, \mathrm{Y}=\mathrm{S}$

Esquema 2. Estructura química de varios compuestos derivados de PEDOT.

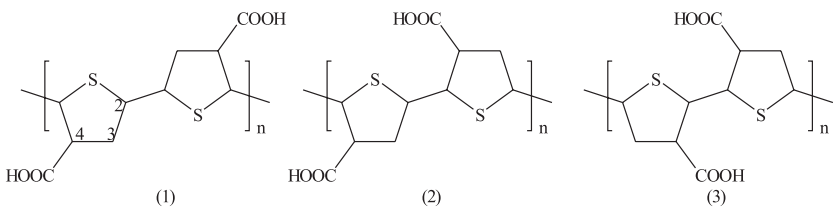

Figura 6. Derivados del 2,2'-politiofeno con grupos carboxílicos en las posiciones 3 y 4 de los anillos de tiofeno.

posiciones originales de los átomos de $\mathrm{S}$ y $\mathrm{O}$, puede tener propiedades eléctricas prometedoras.

Otro campo de investigación importante se centra en cómo mejorar la solubilidad de polímeros derivados del politiofeno, ya que algunas aplicaciones técnicas así lo requieren. Una forma de aumentar la solubilidad en disolventes orgánicos se basa en incorporar determinados grupos funcionales laterales -como cadenas alquílicas ${ }^{[23]}$, sustituyentes ionizables (sulfonatos o carboxilatos), alcoholes o ésteres ${ }^{[24]}$ - a la cadena principal del politiofeno. Sin embargo, la anexión de los grupos laterales puede introducir efectos estéricos que reducen la planaridad de la cadena principal, disminuyendo la conjugación $\pi-\pi$, o puede alterar de forma indeseable otras propiedades del polímero. Por otra parte, es importante conocer la posición más favorable en la que introducir dichos grupos, así como la tacticidad que adoptará el polímero.

Afortunadamente, los problemas que se acaban de mencionar pueden ser abordados empleando técnicas de modelización molecular. Por ejemplo, la introducción de un grupo carboxílico por anillo de tiofeno puede dar lugar a varios compuestos, como los indicados en la Figura 6, cuyas características estructurales y electrónicas pueden ser evaluadas y comparadas desde un punto de vista teórico.

La modelización de estos tres compuestos indica que el derivado (1), con los grupos carboxílicos en la posición 4 de los anillos de tiofeno, es el más estable y adopta una conformación próxima a la planaridad ${ }^{[25]}$. Con relación al valor del band gap, la Figura 7 muestra la variación que experimenta el valor teórico de $\mathrm{E}_{\mathrm{g}}$, obtenido a nivel B3PW91 $(6-31+G(d, p)$, a medida que aumenta el número de anillos de tiofeno que se incluyen en la modelización. Como se puede observar, conforme aumenta el número de anillos, Eg converge hacia un valor similar al del politiofeno. Este resultado sugiere que ambos compuestos neutros tienen una conductividad eléctrica similar.

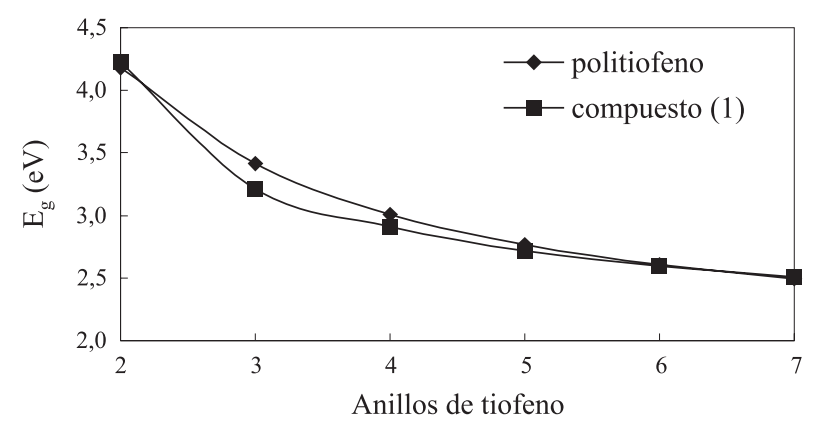

Figura 7. Valor de $\mathrm{E}_{\mathrm{g}}$ obtenido en modelos de politiofeno y del compuesto (1) con varios anillos de tiofeno. 


\section{Conclusiones}

En este artículo se han revisado varias características de algunos polímeros heterocíclicos que están relacionadas con su capacidad para conducir la corriente eléctrica. Los polímeros deben poseer una estructura prácticamente plana y, lógicamente, un valor del band gap pequeño. Por otra parte, bajos potenciales de ionización y altas afinidades electrónicas facilitan el dopado del polímero. Los resultados expuestos en este artículo demuestran que las técnicas teóricas de modelización molecular son adecuadas para evaluar éstas y otras propiedades de manera rápida y relativamente sencilla, a la vez que permiten comprender mejor el fenómeno de la conducción eléctrica.

Por otra parte, es importante destacar que las herramientas de modelización molecular pueden ser aplicadas, al menos en principio, para predecir las propiedades de cualquier compuesto, aunque éste no haya sido nunca sintetizado. En consecuencia, la colaboración entre los laboratorios teóricos y experimentales implica una optimización de los esfuerzos de éstos últimos, que pueden reducir de forma considerable su consumo de recursos humanos e instrumentales.

Todo ello nos lleva a pensar que la modelización molecular contribuirá de forma destacada a la predicción de nuevos candidatos para la próxima generación de polímeros conductores y, en definitiva, a la obtención y al desarrollo de las aplicaciones de los polímeros conductores de este siglo XXI.

\section{Agradecimientos}

Parte del trabajo expuesto o referenciado en este artículo ha sido realizado gracias al proyecto MAT2003-00251 subvencionado por Ministerio de Ciencia y Tecnología.

\section{Referencias}

1. (a) Sirakawa, H.; Louis, E. J.; MacDiarmid, A.G.; Chiang, C.K.; Heeger, A.J. - J. Chem. Soc. Chem. Commun. 578 (1977); (b) Chiang, C.K.; Fincher, C.R.; Park, Y.W.; Heeger, A.J.; Shirakawa, H.; Louis, E. J.; Gao, S.C.; McDiarmid, A.G. - Phys. Rev. Lett 39 1098, (1977).

2. (a) J. Roncali, Chem. Rev. 97 (1997) 173; (b) J. Roncali, Chem. Rev. 92, 711, (1992).

3. "Handbook of conducting polymers" 2 nd ed. (eds: T.A. Skoteheim, R.L. Elsenbaumer, J.R. Reynolds), Dekker, New York, (1998).

4. (a) Frommer, J.E. \& Chance, R.R. - en "Electrical and electronic properties of polymers: a state-of-the-art compendium", (ed: J.I. Kroschwitz), John Wiley, New York, (1988); (b) Chan, H.S.O. \& Choon, S. - Prog. Polym. Sci. 23 (1998) 1167; (c) Martín, R.E.\& Diederich, F. - Angew. Chem., Int. Ed. 38, 1350, (1999); (d) T. Yamamoto, Macromol. Rapid Commun. 23 (2002) 583.

5. Armelin, E.; Iribarren, J.I.; Casanovas, J.; Liesa, F. \& C. Alemán - Química e Indústria 51, 13-20, (2004).

6. Brédas, J.L. \& Street, G.B. - Acc. Chem. Res. 18, 309, (1985).
7. Furukawa, Y. - J. Phys. Chem. 100, 15644, (1996).

8. Brédas, J.L.; Thémans, B.; Fripiat, J.G. \& André, J.M. - Phys. Rev. B 29, 6761, (1984).

9. Schmidt, W.; Baldridge, K.K.; Boatz, J.A.; Elbert, S.T.; Gordon, M.S.; Jensen, J.H.; Koseki, S.; Matsunaga, N.; Nguyen, K.A.; Su, S.J.; Windus, T.L.; Dupuis, M. \& Montgomery, J.A. - J. Comput. Chem. 14, 1347. PC-GAMESS supplied by A. Granovsky, Moscow State University, (1993).

10. Gaussian 98, Revision A.7, M.J. Frisch et al., Gaussian, Inc., Pittsburgh PA, 1998.

11. Raos, G.; Famulari, A. \& Marcon, V. - Chem. Phys. Lett. 379, 364, (2003).

12. Alemán, C. \& Julià, L. - J. Phys. Chem. 100, 1524, (1996).

13. Alemán, C.; Domingo, V.M.; Fajarí, L. \& Julià, L. - J. Org. Chem. 63, 1041, (1998).

14. Alemán, C.; Armelin, E.; Iribarren, J.I.; Liesa, F.; Laso, M. and Casanovas, J. - Synthetic Metals, 149, 151, (2005).

15. Becke, A.D. - J. Chem. Phys. 98, 1372, (1993); Perdew, J.P. \& Wang, Y. - Phys. Rev. 45, 13244, (1992).

16. Casanovas, J. \& Alemán, C. - resultados no publicados.

17. Almenningen, A.; Gastiansen, O. \& Suendsas, P. - Acta Chem. Scand. 12, 1671, (1958).

18. Koopmans, T. - Physica 1, 104, (1934).

19. (a) Alemán, C.; Casanovas, J. - J. Phys. Chem. A 108, 1440, (2004); (b) Casanovas, J.; Ricart, J.M.; Rubio, J.; Illas, F. Jiménez-Mateos, J.M. - J. Am. Chem. Soc. 118, 8071, (1996); (c) Bagus, P.S.; Illas, F., Casanovas, J.; Jiménez-Mateos, J.M. - J. Electron. Spectroc. 83, 151, (1997).

20. Alemán, C. \& Julià, L. - J. Phys. Chem. 100, 14661, (1996).

21. Alemán, C. - Macromol. Theory Simul. 6, 237, (1997).

22. Alemán, C.; Curcó, D. \& Casanovas, J. - Chem. Phys. Lett. 386, 408, (2004).

23. (a) Miller, G. G.; Elsenbaumer, R. L. - J. Chem. Soc., Chem. Commun. 169 (1986); (b) Garnier, F. - Angew. Chem., Int. Ed. Engl. 28, 513 (1989); (c) Chen, T.A.; Wu, X.; Rieke, R.D. - J. Am. Chem. Soc. 117, 233, (1995); (d) Roncali, J.; Garreau, R.; Delabouglise, D.; Garnier, F.; Lemaire, M. Synth. Met. 28, C341, (1989); (e) Souto Maior, R.M.; Hinkelman, K.; Eckert, H.; Wudl, F. - Macromolecules 23, 1268, (1990); (f) Bartlett, P.N.; Dawson, D.H. - J. Mater. Chem. 4, 1805, (1994); (g) Demanze, F.; Yassar, A.; Garnier, F. - Adv. Mater. 7 ( 1995) 907.

24. (a) Kim, B.; Chen, L.; Gong, J.; Osada, Y. - Macromolecules 32, 3964, (1999); (b) Rasmussen, S.C.; Pickens, J.C.; Hutchison, J.E. - Macromolecules 31,933,(1998); (c) Kim, Y.-G.; Samuelson, L.A.; Kumar, J.; Tripathy, S.K. - J. Macromol. Sci. A39, 1127, (2002); (d) Rasmussen, S.C.; Pickens, J.C.; Hutchison, J.E. Chem. Mater. 10, 1990, (1998); (e) Kim, B.-S.; Fukuoka, H.; Gong, J.P.; Osada, Y. - Eur. Polym. J. 37, 2499, (2001); (f) Masuda, H.; Kaeriyama, K. - Makromol. Chem., Rapid Commun. 13, 461, (1992); (g) McCullough, R.D.; Ewbank, P.C.; Loewe, R.S. - J. Am. Chem. Soc. 119, 633, (1997).

25. Casanovas, J.; Zanuy, D. \& Alemán, C. - Polymer 46, 9452 (2005).

Enviado: 10/02/05

Reenviado:03/05/05

Aprovado: 13/05/05 